cross-sectional rather than longitudinal data. Methods: We analyzed 3MS data from English speakers in the CSHA for DIF with respect to gender, birth cohort, and education. We initially focused on cross-sectional data from participants who had data at all three evaluations $(n=2,365)$. We performed cross-sectional analyses using a hybrid ordinal logistic regression / item response theory (OLR/IRT) framework for DIF detection for each covariate at each evaluation. For longitudinal analyses on this data set and on the entire data set of English speakers $(n=7,221)$, we modified the OLR/IRT framework to account for covariation within individuals over time by clustering on person. We used this framework to determine person-level and group-level DIF impact. With the longitudinal data we also determined whether accounting for DIF impacted the estimated rate of cognitive decline for each subgroup. Results: Cross-sectional item-level findings differed at different time points. Accounting for DIF related to gender, birth cohort, and education had a salient impact on sizable numbers of individuals, ranging from $4 \%$ to $13 \%$ at each time point. Item-level results from the longitudinal DIF framework were broadly consistent with the cross-sectional results, though there was more power with the longitudinal data. Accounting for DIF increased the difference in rates of cognitive decline between those with high and low education from -0.02 to -0.05 SD over five years; ignoring DIF resulted in modestly under-estimating the rate of decline for those with lower education. Conclusions: Differences between groups in rates of cognitive decline may in part be due to measurement problems such as DIF, as shown here for education. Studies evaluating factors associated with cognitive decline should account for DIF to reduce Type I error risk due to DIF. The framework illustrated here is a useful starting place for further methods development.

\section{P3-071 DEMENTIA AND TYPE 2 DIABETES: THE ROLE OF BODY COMPOSITION AND INSULIN DEGRADING ENZYME}

Mary N. Haan ${ }^{1}$, Pattie Green ${ }^{2}$, Suzanne Craft ${ }^{2}$, Adina Zeki Al-Hazzouri ${ }^{3}$, Rachel Whitmer ${ }^{4},{ }^{1} U C S F$, San Francisco, CA, USA; ${ }^{2}$ University of Washington, Seattle, WA, USA, ${ }^{3}$ University of Michigan, Ann Arbor, MI, USA;

${ }^{4}$ Kaiser Division of Research, Oakland, CA, USA. Contact e-mail: mary. haan@ucsf.edu

Background: Type 2 diabetes is associated with a greater risk of Alzheimer's disease [AD], and dementia. Insulin degrading enzyme [IDE] regulates insulin and may link type 2 diabetes and dementia. Lean body mass declines are linked to increases in insulin resistance. This study evaluates the role of IDE as a pathway between body composition and dementia. Methods: A subsample $(n=819 / 1789)$ of participants from the Sacramento Area Latino Study on Aging, was classified as: demented [ $\mathrm{n}=$ 39], diabetic [ $\mathrm{n}=280]$, both diabetic and demented $[\mathrm{n}=56]$ or neither $[\mathrm{n}=444]$. IDE was analyzed from whole blood using a measure of immunoreactivity that quantified relative enzyme levels. Body fat (BF) and lean mass (LBM) were analyzed with bioelectric impedance. Biomarkers included IL-6, TNF- $\alpha$, C-reactive protein, insulin sensitivity (QUICKI), glucose, leptin and adiponectin. Correlations between IDE and each of these biomarkers were analyzed overall and by diagnostic group. Polytomous logistic regression models evaluated the association between IDE and the three diagnoses compared to no diagnosis. Covariates included age, education, nativity and all biomarkers. Results: Among women with neither diagnosis, IDE $(\log )$ was correlated with glucose $(r=0.12)$, insulin $(r=0.12)$, LBM $(r=0.16)$, QUICKI $(r=-0.12)$, and lean body mass $(r=0.16)$. Among women with only dementia/CIND, higher IDE $(\log )$ was correlated with lower IL-6 and TNF- $\alpha$. Higher IDE $(\log )$ was associated with a lower risk of having both diabetes and dementia $(\mathrm{OR}=0.42,95 \% \mathrm{CI}$ : $0.22-0.82$, $\mathrm{p}=0.01)$, even after adjustment for all covariates but IDE(log) was not associated with dementia or diabetes alone. LBM moderated the association between IDE $(\log )$ and 'both' diagnoses such that a 1 SD higher LBM was linked to a $40 \%$ lower risk of both diagnosis from IDE( $\log )$ ( $\mathrm{p}$ for $\mathrm{IDE}^{*} \mathrm{LBM}$ interaction $=0.001$ ) compared to no diagnosis. Among men, IDE $(\log )$ was not correlated with any biomarker or associated with any diagnosis. In both genders, LBM was lowest in demented cases, and highest in diabetics. Conclusions: In women, IDE is linked to inflammation and metabolic markers. IDE $(\log )$ is more protective for combined diagnosis in women with higher lean body mass. These findings suggest that lower LBM may be a pathway by which IDE may increase the risk of dementia.

\section{P3-072 DIFFERENCES IN AGE, GENDER, EDUCATION AND COGNITIVE STATUS IN PATIENTS WITH DEMENTIA IN THE TWO LAST DECADES}

M. Tsintou ${ }^{1}$, M. Tsolaki ${ }^{2}$, D. Liapi ${ }^{1}$, F. Kounti ${ }^{3}$, N. Vlaikidis ${ }^{4},{ }^{1}$ Greek Alzheimer's Disease and Related Disorders Association, Thessaloniki, Greece; ${ }^{2} 3$ rd Department of Neurology, Aristotle University of Thessaloniki; Greek Alzheimer's Disease and Related Disorders Association, Thessaloniki, Greece; ${ }^{3}$ Greek Alzheimer's Disease and Related Disorders Association, Thessaloniki, Greece; ${ }^{4} 3$ rd Department of Neurology, Aristotle University of Thessaloniki, Thessaloniki, Greece. Contact e-mail: magda. tsintou@gmail.com

Background: The aim of this study is to examine the first Mini-Mental State Examination (MMSE), widely used in Greece, education, age and gender of patients with dementia examined during the 1990-1999 and 2000-2009 decades and discuss the possible differences. Methods: A 2583 representative sample of patients with cognitive decline was examined at outpatient Memory and dementia Clinic of $3^{\text {rd }}$ University Department of Neurology and Alzheimer's Disease Center of Greek Alzheimer Association. Results: From the whole sample $46.3 \%$ were examined in 1990-1999 decade, (the $41.7 \%$ of which were men and $58.3 \%$ women) and $53.7 \%$ were examined in $2000-2009$ decade (the $27.4 \%$ of which were men and $72.6 \%$ women). The MMSE and the educational years' means of the 1990-2000, according to the SPSS software independent ttest, were $21.09 \pm 7.173$ and $6.91 \pm 4.278$ respectively and the 20002009 means were $24.03 \pm 6.009$ and $9.01 \pm 4.655$ respectively. The 2000-2009 MMSE, education years and patients' age means, being $24.03 \pm 6.009,9.01 \pm 4.655$ and $69.67 \pm 10.037$ respectively, were greater than the 1990-1999 ones. Comparing patients equally educated, a statistically significant patients' MMSE increase $(p=0<0.01)$ was found for 2000-2009 in relation to the 1990-1999 patients' MMSE. That means that patients visit earlier the Dementia Centers. Despite the greater men's education years mean $(8.59 \pm 4.869)$ than the women's one $(7.76 \pm 4.437)$, the men's age mean $(68.03 \pm 11.976)$ is greater than the women's $(66.90 \pm 11.823)$ and men's MMSE (22.53 \pm $6.728)$ is almost equal to the women's $(22.74 \pm 6.738)$. Contrary to the 2000-2009 results, in which men had not statistically significantly higher MMSE results than women $(\mathrm{p}=0.131>0.05)$, higher MMSE results were taken from women during 1990-1999, being statistically significant $(\mathrm{p}=$ $0<0.01)$. The differences found between the examination age $(\mathrm{p}=$ $0<0.01)$, the MMSE $(\mathrm{p}=0<0.01)$ and the educational level $(\mathrm{p}=$ $0<0.01$ ) in both decades were statistically significant. Conclusions: During the decade 2000-2009 more patients visited our centers earlier (MMSE better), more women than men, less educated, and younger than in decade 1990-1999. We believe that awareness and increase of mean survival time could explain these results.

\section{P3-073 FUNCTIONAL BIOMARKERS AS PREDICTORS OF CONVERSION FROM NORMAL COGNITIVE FUNCTION TO PRE-CLINICAL DEMENTIA SYNDROMES: THE PATH THROUGH LIFE STUDY}

Kaarin J. Anstey, Nicolas Cherbuin, Australian National University, Canberra, Australia.Contacte-mail:kaarin.anstey@anu.edu.au

Background: Functional biomarkers were initially developed as indices of biological ageing. They have been linked to cognitive decline, mortality, and falls and to intra-individual variability in reaction-time which is a marker of brain damage and found to be increased in dementia, MCI, and ageing. 